\title{
A Study on the Distortion of Course Information due to Wheel- shaped Barbed-wires Fences
}

\author{
Jeongil Moon $^{1}$ and Yoonsik Kwak ${ }^{2}$ \\ ${ }^{1}$ Civil Aviation Training Center, Korea Airport Corporation, \\ ${ }^{2}$ Department of Computer Engineering, Korea National University of \\ Transportation, \\ 'ynhae@airport.co.kr, ${ }^{2} y s k w a k @ u t . a c . k r$
}

\begin{abstract}
The very high frequency (VHF) Omnirange is used as an aircraft navigational aid. The VHF Omnirange is a short-range air navigation system providing aircraft with its bearing relative to the ground station. The VHF Omnirange has undergone a number of evolutionary changes in the past 60 years. The objectives of the improved VHF Omnirange would be a system that would significantly reduce the magnitude of bearing errors, provide greater accuracy and use digital techniques to improve the system. The VHF Omnirange signal in space is a composite of the direct and scattered carrier plus sideband and identification signals. The receiver antenna is designed to pick up only the horizontally polarized component of the electric field available at the antenna.

The bearing errors caused by the obstacles have been examined by Anderson, Gruenberg and Yamamoto. This paper quoted the research with the principle theory such as mathematical analysis regarding scalloping errors. The objective of this paper is to derive an analysis method for quantitative prediction of the distortion of course information due to the wheel-shaped wires. The main emphasis is the error caused by the scattered signal at actual site. Course scalloping is a periodic variation of the indicated bearing about the correct azimuth, where the frequency varies with position in space. The results reported in this paper were obtained by mathematical analysis, computer-based simulations and experimentation.
\end{abstract}

Keywords: Very high frequency Omnirange, Navigation Aids

\section{Introduction}

The VHF Omnirange is a navigation support system that provides directional information to aircraft. The ground station gives the azimuth information using the two $30 \mathrm{~Hz}$ signal. The VHF Omnirange receiver employs a phase comparator to compare the phases of two $30 \mathrm{~Hz}$ audio signals. Since aircraft receiver is likely to be affected by the surrounding geography and buildings, mountains. The aircraft received at $\mathrm{P}$ (in figure1) is combination of the carrier wave and the sideband. The signal at the receiving point is a combination of errors. The VOR signal including the effects of the three error sources is a combination. In addition to the foregoing, there is an error caused by the interference of the original sideband with the re-radiated sideband from the carrier antenna. However, the waveform distortion of the sideband due to the interference and the effect of the distributor, the counterpoise is neglected here. To evaluate the accuracy, an experimental test station is used for actual measurement of the error which compared with the predicted results. 


\section{Operating Principle}

Figure 1 shows the antenna system explaining the operating principle of VOR. The antenna system consists of the antenna Ac radiating the carrier wave containing the reference phase signal and the upper and lower sideband antennas rotating on the circle of radius $r$ by 30 times per second for radiating the variable phase signals.

The carrier wave at the point $\mathrm{P}$ is located at angle with the magnetic north axis and a distance $\mathrm{d}$ from point $\mathrm{A}$ can be given as follows

$$
E_{\text {carr }}=\cos \left(w_{1} t-\beta_{1} R\right)(1+m \omega s \rho t)
$$

where $\beta_{1}=\frac{2 \pi}{\lambda_{1}}, \mathrm{~m}$ is the modulation index and is $0.3, f_{1}=w_{1} / 2 \pi$ is the carrier frequency.

$$
\Delta f=\frac{v f}{c}= \pm 480 H z=r \times \frac{\pi f_{m}}{\lambda}=13.45(m) \times \frac{30 \pi}{2.64}
$$

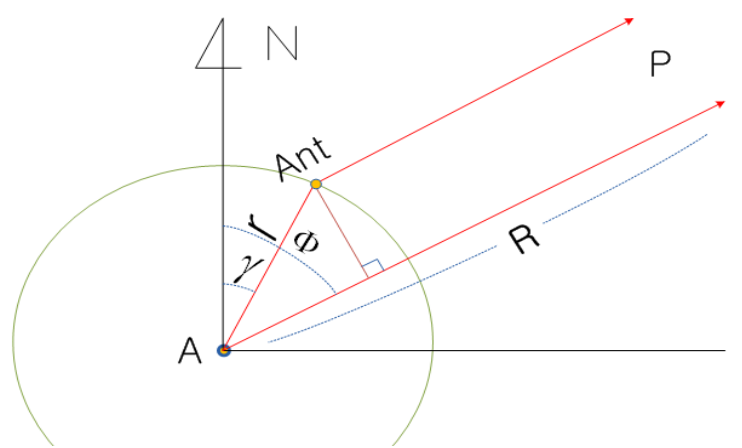

Figure 1. VOR Antenna Principle

where ; $\Phi$ is Azimuth angle regarding aircraft, רis Azimuth angle of antenna On the other hand, the sideband $E_{s}$ tis given as follows:

$$
E_{s b}=\cos \left[w_{2} t+\beta_{2} \operatorname{ros}(\Phi-\rho t)-\beta_{2} R_{\text {. }}^{-}\right.
$$

where $\beta_{2}=2 \pi / \lambda^{2}$, electrically rotating frequency of antennas $f_{2}=w_{2} / 2 \pi$

We can differentiate the frequency in oder to get the instance frequency $f_{\text {inst. }}$.

$$
f_{\text {inst }}=\frac{1}{2 \pi} \frac{d}{d t}\left[\omega_{2} t+\beta_{2} r \cos (\Phi-\rho t)-\beta_{2} R\right]=f_{\mathbf{z}}+\frac{r \rho}{\lambda} \sin (\Phi-o t)
$$

Consider a transmitter and a receiver spaced apart by $\mathrm{D}$ meters. The number of wavelengths between the receiver and transmitter is $D / \lambda$. Expressed as a phase difference this is $\Phi=2 \pi \mathrm{d} / \lambda$ radians. When the receiver and transmitter are moving with respect to one another, $\Phi$ is changing and a change in phase corresponds to a change in frequency or a Doppler shift.

Doppler shift is $\Delta f=\frac{1}{2 \pi} \frac{d \Phi}{d t}=\frac{1}{2 \pi} \frac{2 \pi}{\lambda} \frac{d D}{d t}=\frac{1}{\lambda} \frac{d D}{d t}=\frac{1}{\lambda} \cdot{ }_{\text {(relative velocity) }}=V_{r} \frac{f_{0}}{c}$ where; $V r$ is the velocity of the receiver relative to the transmitter

$f_{\text {ois }}$ the transmitter frequency and $\mathrm{c}$ is the velocity of light

The received frequency will therefore be above, below or equal to $\mathrm{fc}+9960 \mathrm{~Hz}$ is proportional to the product of "D" and rpm. Since the frequency modulation deviation cycle must occur once every 30 second, rpm is fixed at $30 \mathrm{~Hz}$. 
The diameter of the aerial ring directly affects the deviation ratio of the $9960 \mathrm{~Hz}$ sideband as shown in the following formula.

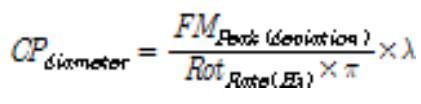

The FM Deviation provided by the VOR is based on the Doppler principle. It is a fact that an observer will experience an increase in frequency as an RF source moves towards them and a decrease in frequency when the RF source moves away. The amount of frequency change is related to the velocity of the RF source towards/away from the observer.

The VOR receiver does not react to the sideband at $60 \mathrm{~Hz}$ and above because the VAR signal is separated by a low pass filter.

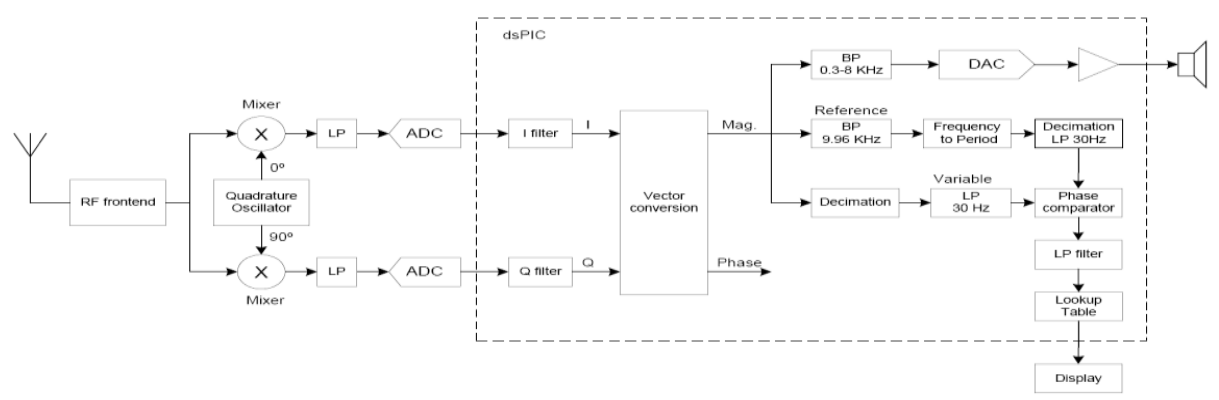

Figure 2. Block Diagram of a Receiver Unit

Figure 2 represents the receiver of the VOR. A receiver in the aircraft receives the sum of all modulation components from the ground station. A filter separates the $30 \mathrm{~Hz} \mathrm{AM}$ component of the rotating beam from other modulation component of the rotating beam from other modulation components. Likewise, a filter selects the $9960 \mathrm{~Hz} \mathrm{FM}$ component. The FM signal is then detected to recover the $30 \mathrm{~Hz}$ reference signal, which in turn is compared in phase with the signal from the rotating beam. An essential requirement for the separate carrier and sideband signals to combine correctly in the air is that the two sideband and the carrier have the correct phase relationship. If the carrier and the sideband signals are treated as three rotating vectors, then the upper sideband having the highest frequency, will rotate fastest. The carrier will rotate next fastest and the lower sideband will be the slowest.

\section{Distortion of Course Information}

The total signal combining $E_{c a r r}$ and $E_{s}$ is incident in the receiver. Since the detected

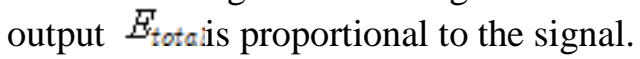

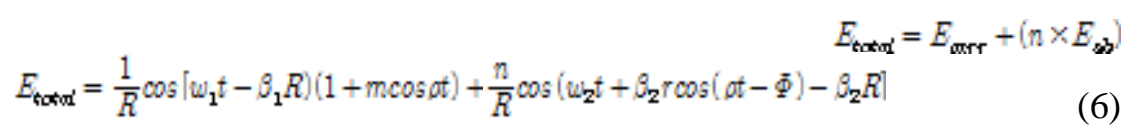

Where the second part is the Reference phase part and the 3rd component is the second carrier component modulated by the $30 \mathrm{~Hz}$ variable phase frequency. Because this 2 nd carrier frequency is frequency modulation demodulated, the $30 \mathrm{~Hz}$ variable phase part is received. A block diagram of the aircraft receiver received the signal at the antenna. The output of the first detector will be the envelope of the $E_{\text {tota:signal. }}$ 


$$
E_{\text {tota: }}=1+m \cos \left(\omega_{2} t+m, \sin \sigma t\right)+n\left[\cos \left(\omega_{2} t-\Phi\right)-0.0445 \cos \left(\omega_{2} t+3 \Phi\right)\right]
$$

The second term passes via the $9960 \mathrm{~Hz}$ BPF.

Generally FM signal is $f_{i}(t)=f_{i}+k_{i} m(t)$ and this signal is differentiated by the device function $H(f)=j 2 \pi f=2 \pi|f| \angle 90^{\circ}$ therefore the output should be

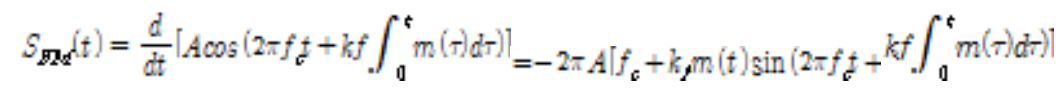

The detected envelope signal is $2 \pi A\left[f_{\mathrm{v}}+k f m(t)\right]$ and differentiate the 2 nd term of (6)

$$
\frac{d}{d t}(m, \sin \alpha t)=a m_{,} \cos \alpha t
$$

This is the variable phase $30 \mathrm{~Hz}$. The third term in (7) $" \cos \left(w_{2} t-\Phi\right) "$ is the passes the Reference $30 \mathrm{~Hz}$ BPF.

If an wheel-shaped barbed-wires are located close to the antenna system, it will affect the original radio patterns. The scattered electronic field in space affect the angular distributions. The distortion radio pattern $E_{\text {Sctcan be presented below }}$

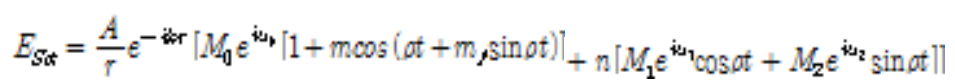

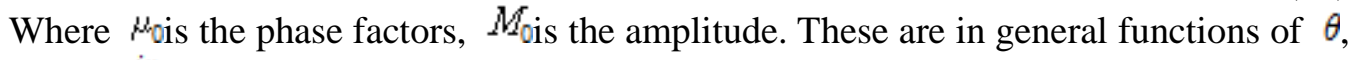
$\Phi$ and $M_{0} e^{i \mu_{k}}$ can be obtained by an incident field with $\mathrm{A}=1$. The scattered field for the side bands must be computed for $A_{n}=1 . M_{i}$ and $\mu_{i}$ must be determined with the modulation factors omitted and must include the effect of ground images.

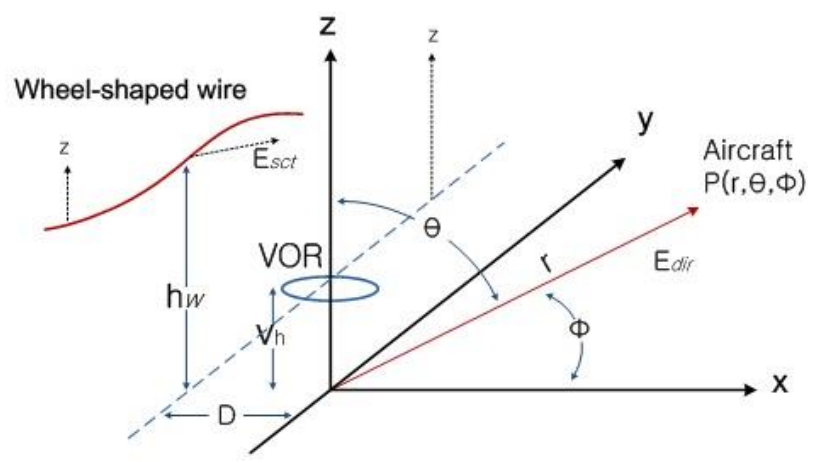

Figure 3. Rectangular Coordination System

The phases in the 3 terms must reflect the fact that all scattering patterns must be referred to the origin as the phase center. The total field at the aircraft is the sum of $E_{d \mathrm{dr}}$ and $E_{\text {sct. }}$ It has components in phase and in quadrature with the direct carrier field $E_{d \mathrm{~d} \text { r. }}$ These 2 components are seen to be proportional to

$$
\begin{gathered}
{\left[1+\left(M_{0} / S\right) \cos \mu_{0}\right]\left(1+m \cos \left(o t+m_{f} \sin \rho t\right)\right.} \\
1+n\left[\cos \Phi+\left(\frac{M_{1}}{S}\right) \cos \mu_{1}\right] \cos \rho t 1+n\left[\sin \Phi+\left(\frac{M_{2}}{S}\right) \cos \mu_{2}\right] \sin \rho t \\
\left.\left(M_{0} / S\right) \sin \mu_{0}\right]\left(1+m \cos \left(o t+m_{f} \sin \rho t\right)+n\left[\left(\frac{M_{1}}{S}\right) \sin \mu_{1} \cos \rho t+\left(\frac{M_{2}}{S}\right) \sin \mu_{2} \sin o t\right]\right.
\end{gathered}
$$

In practice the terms involving $\left.M_{\mathrm{f}} / \mathcal{E}_{(\text {for }} i=0,1,2\right)$ will be small compared to unity. 
This will even be the case as $\theta$ tends to "0" because the scattering amplitudes $M_{\text {:must }}$ contain a factor $\sin \left(k h_{\psi} \beta\right)$, where $h_{w i s}$ the height of the wire obstacles above ground. This factor arises from the image in the ground.

The $\left.M_{4} / \varepsilon_{(\text {for }} i=0,1,2\right)$ consists a factor $\sin \left(k h_{\psi} \theta\right) / \sin \left(k h_{v} \theta\right)$ which remains bounded as $\theta$ tends to zero. If terms of order $\left(M_{\mathrm{f}} / S\right)^{z}$ are neglected, the output of the first envelope detector will be proportional to the in phase component given by (11). The first term of the formular (11) will be processed by the $9960 \mathrm{~Hz}$ Band pass filter and the discriminator, and lead to the zero phase reference signal.

$$
\frac{\sin \Phi+\left(M_{2} / S\right) \cos \omega_{2}}{\cos \Phi+\left(M_{1} / S\right) \cos \omega_{1}}=\tan (\Phi+\Delta \Phi)
$$

$(\Phi+\Delta \Phi)$ is the phase comparator. If the bearing error $\Delta \Phi$ exceeds several degrees in some azimuthal sector, that sector will in general present navigational difficulties.

One may therefore assume that $\Delta \Phi<<$ land one obtains are some algebra:

$$
\begin{aligned}
\Delta \Phi=[ & {\left.\left[\frac{M_{2}}{S}\right) \cos \Phi \cos \omega_{z}-\left(\frac{M_{1}}{S}\right) \sin \Phi \cos \omega_{1}\right] /\left[1+\left(\frac{M_{1}}{S}\right) \cos \Phi \cos \omega_{1}+\left(\frac{M_{2}}{S}\right) \sin \Phi \cos \omega_{2}\right] } \\
& \cong\left(\frac{1}{S}\right)\left(M_{z} \cos \Phi \cos \omega_{2}-M_{1} \sin \Phi \cos \omega_{1}\right)
\end{aligned}
$$

Where $\cos \mu_{\text {and }} \cos \mu_{\text {zare }}$ fairly quickly changeable functions of $\Phi$. This cannot bear comparison with other factors in (14). It is difficult to estimate in general without some

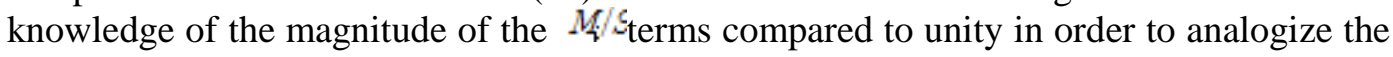
error in the final stages. Possibly it can be shown that error is less than 10 percent in most realistic circumstances from the sturdy of extraordinary occasion. The second item has the advantage that it permits a simple physical description of the nature of bearing error. In consideration of a small scatter in the vicinity of antenna system at distance D, No generality is lost by locating the scatter in the plane $\Phi=0$. It is then only illuminated by the even sideband because the odd sideband has a null in the direction. One can always turn round the coordinate system and translate the time scale to get the same circumstance at one time. For this circumstance, (14) reduced to

$$
\Delta \Phi=-\left(\frac{M_{1}}{S}\right) \sin \Phi \cos \mu_{1}
$$

It is easy to see from figure 2 that the phase term in the scattered field pattern must be the form

$$
\mu_{1}=k D \cos \Phi+\alpha_{1}(\Phi)
$$

In the regions affected by the radio waves are usually cleared of all obstacles within a radius $300 \mathrm{~m}$. Therefor most of the scatterers $k D$ is larger than 360 and $\cos \mu_{1 \text { is a very }}$ quickly oscillating function of $\Phi$. The formula (15) can be viewed as an envelope which is a comparatively smooth function of $\Phi_{\text {which }}$ is broken up into a large number of diffraction lobes. For this reason, the bearing error is often referred to as the scalloping error. The item $\cos \mu$ is called the scalloping item, and the scalloping envelope in degrees is given by

$$
\Delta \Phi_{\text {env }}{ }^{\circ}=\frac{180}{\pi} \mid \frac{M_{1}(\theta, \Phi) \sin \Phi}{S(\theta)}
$$

The scalloping envelope gives the peak bearing error as a function of aircraft bearing. It is a static result which can be obtained by moving a receiver on the ground in a circle around the antenna. If we monitored large circular orbit along a course transverse to the antenna, the receiver will pass quickly via the diffraction radio waves lobes. The monitored signal will change on a regular basis. Because the receiver output is heavily loaded by a capacitor to avoid excessive needle swings, the dynamic error peaks will be 
reduced from the values given by (17). In other words, when the flight inspector flies a near radial course toward or away from the antenna, the scalloping frequency will be low and the dynamic errors will be close to the static values given above. By flying the aircraft along different courses, it is sometimes feasible to determine the location of obstacles.

For scattering obstruction that corresponds to a large angles at the antenna, both sideband scattering patterns should be estimated. Because the $\cos \mu_{\text {and }} \cos \mu_{\text {are }}$ fairly changeable functions of $\Phi$ regarding comparison with other factors in (14), the scalloping envelope will be given by

$$
\Delta \Phi_{\theta n}{ }^{0}=\frac{180}{\pi S(\theta)}\left[\left(M_{2} \cos \Phi\right)^{2}+\left(M_{1} \sin \Phi\right)^{2}-2 M_{1} M_{2} \sin \Phi \cos \Phi \cos \left(\alpha_{2}-\alpha_{1}\right)\right]^{1 / 2}
$$

\section{Simulation Results}

For the purposes of evaluating the present circular barbed-wire, computer software was used to simulate conditions on theory. In the computer simulation, an experimental system with a counterpoise $25 \mathrm{~m}$ in diameter is used at a frequency of $110.6 \mathrm{MHz}$.

Figure 4 shows the test environmental conditions in 3D simulation software. Figure 7 shows the result obtained for the present actual station site which has the similar circumstances with only fences that has a strong resemblance to computerbased simulation1 (Figure 5). Figure 9, 10 shows the result obtained for the present actual station site which has the similar circumstances with circular barbed-wires fences (corresponds to the Figure 6). In the experiment, an experimental station with a $17 \mathrm{~m}$ in diameter counterpoise is used. The obstacles such as fence and circular barbed-wire are $70 \mathrm{~m}$ far away from the antenna system as shown Figure 4.

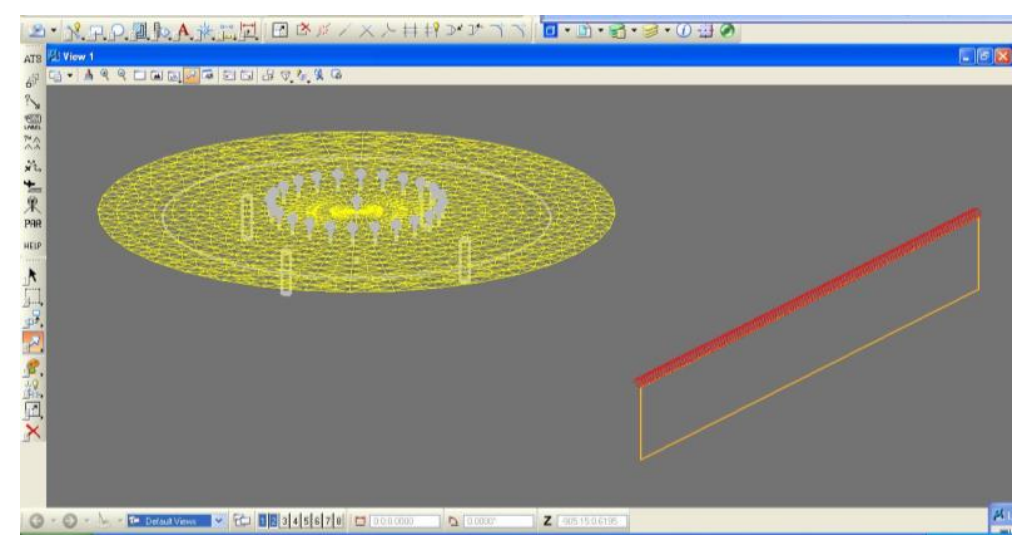

Figure 4. Fence with Circular Barbed-wire Model 


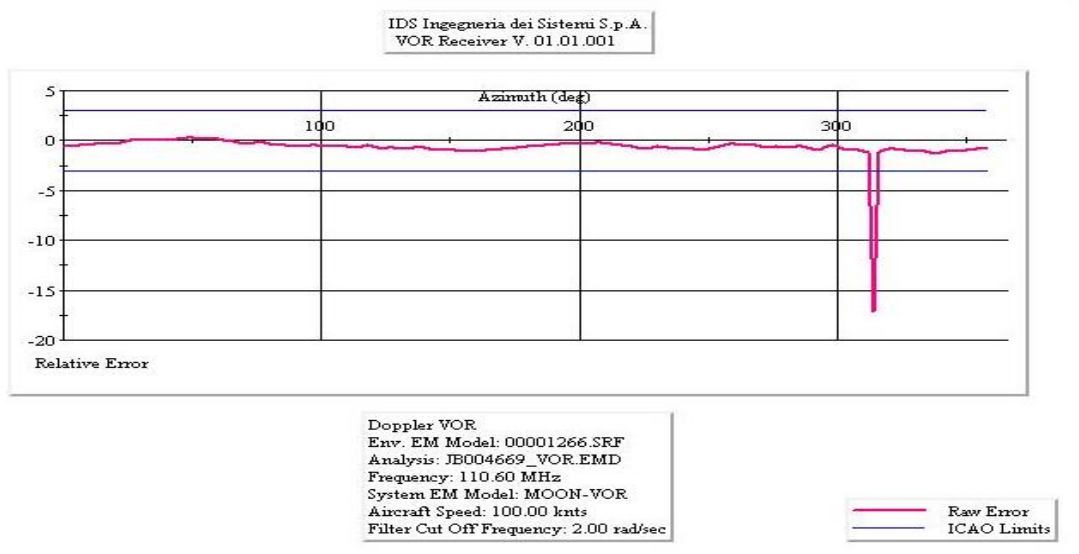

Figure 5. $360^{\circ}$ Error Orbit due to Only Fences (Computer-based Simulation 1)

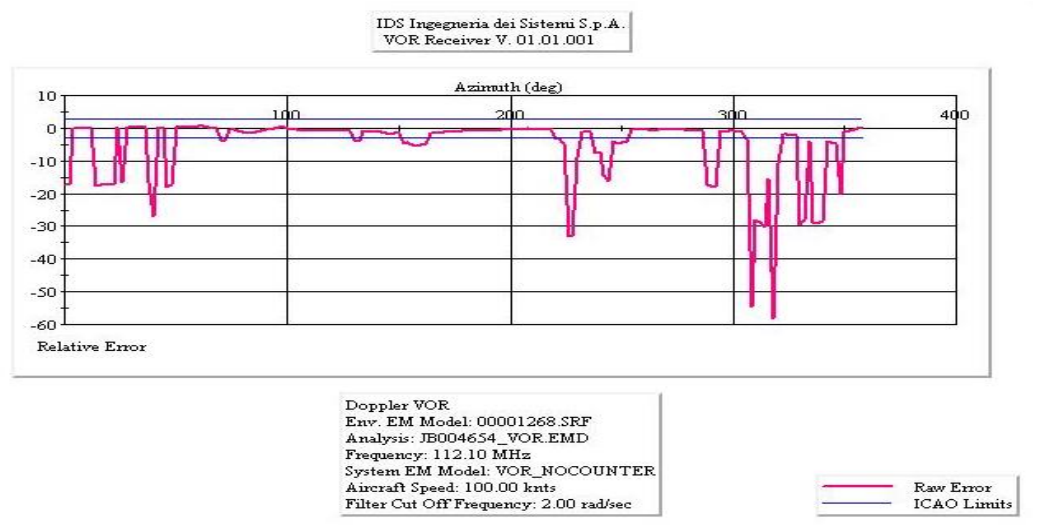

Figure 6. $360^{\circ}$ Error Orbit due to with Circular Barbed-wires Fences (Computer-based Simulation 2)

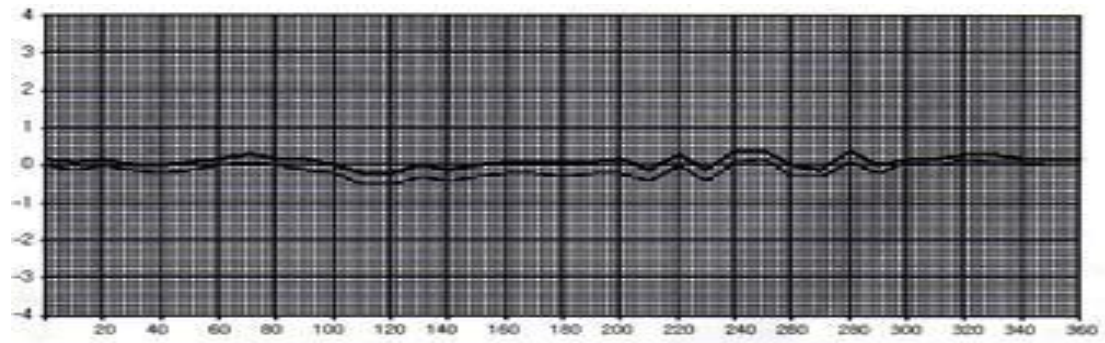

\section{Figure 7. Only Fence Obstacles (Flight Inspector Actual Measurement)}

The first test simulation $360^{\circ}$ error Orbit described were made with the configuration of the only fence without circular barbed-wire. The $360^{\circ}$ error curves show that azimuth angles are influenced by long obstacles such as fences. Single large azimuth errors, due to the fence, would occur. In order to get the information regarding circular barbed-wire effect, we added the wheelshaped barbed-wires to the same condition for the first simulation (Figure 5). This is the second simulation (Figure 6) which was completely changed. Even although we adopted same environment except the extra circular barbed-wire the results was critically changed. 


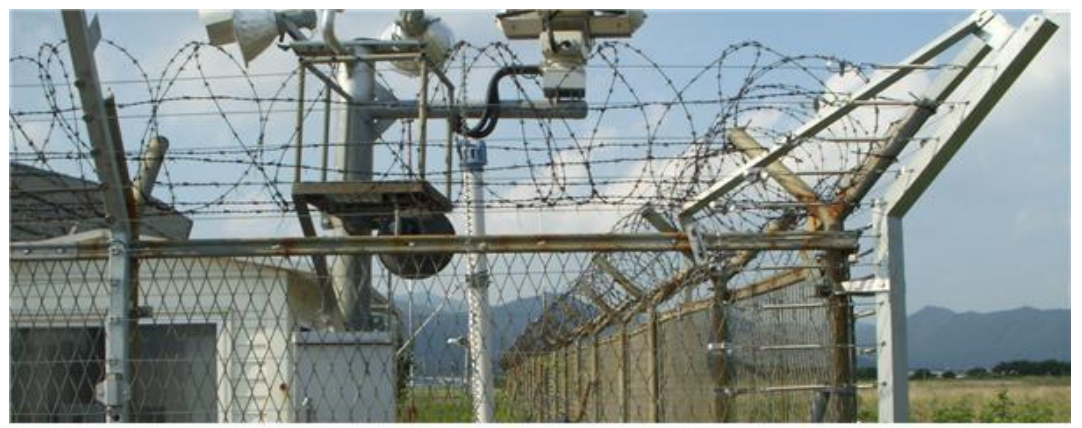

Figure 8. The Circular Twisted Together Wire (Actual Case Site)

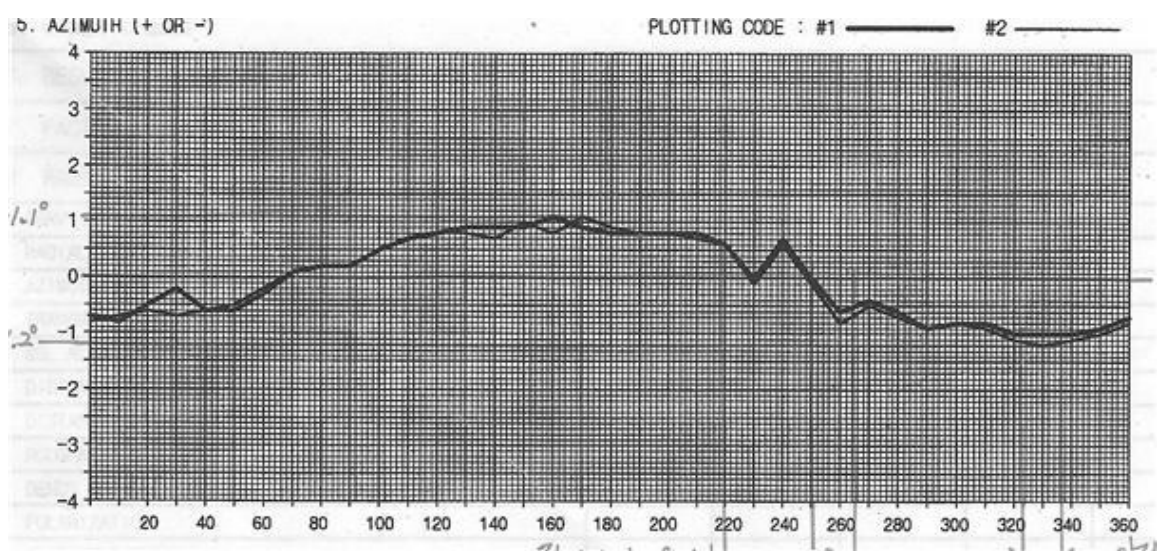

Figure 9. Azimuth Orbit Error_Circular-wires (Flight Inspector Actual Measurement)

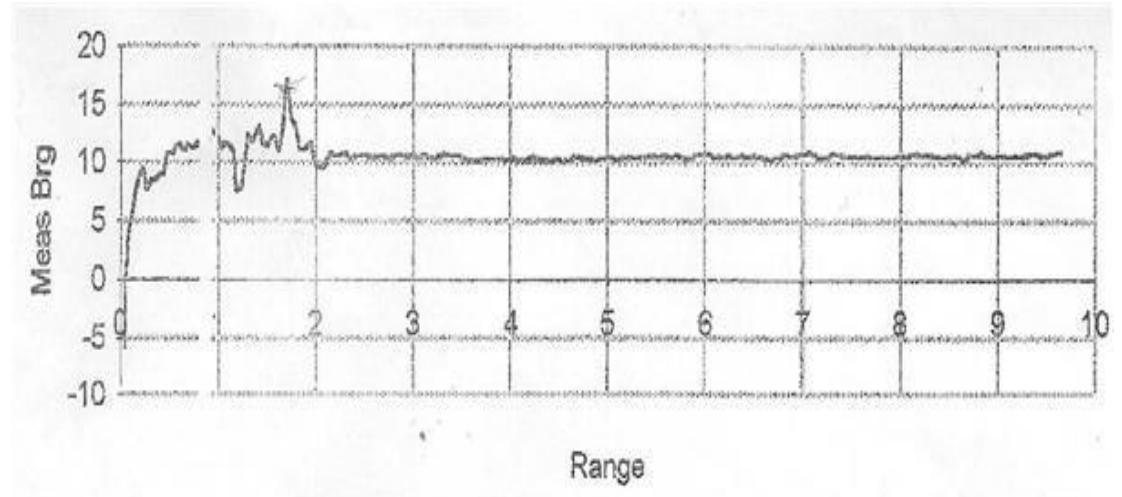

\section{Figure 10. Bearing Error_Circular-wires (Flight Inspector Actual Measurement)}

We experimented real system in order to prove the computer-based simulations (Figure 5, 6). Based on the measured results, the results are shown in Figure 9, 10. The $360^{\circ}$ error curve for an aircraft in orbital flight (Figure 9) and for an aircraft in radial flight (Figure 10) are treated separately. The geometry for a nondirectional re-radiator such as fence with circular wire causing azimuth error is shown in Figures 9 and 10 (corresponds to simulation in the Figure 6).

\section{Conclusions}

This paper points out that complexity of effects of the obstacles and wires was theoretically analyzed. The results were obtained by mathematical analysis, computer-based simulations and experimentation. The theory and computer-based simulations correctly predicts the angular distribution of the azimuth error including the fact that in the event that the fence does not have 
circular barbed-wire, inclined due to the scatters by more than 30 percent sector. There is excellent agreement between theory and experiment on the question of circular barbed-wire.

A number of experiments have been carried out on computer-based simulations in order to evaluate the accuracy with the actual measurement of present station site. An analysis of all the above experimental data indicates that even though two fences are pretty much of the same obstacle, the error was influenced by the scattered radio wave such as circular wire. With respect to course error characteristics, a long circular reflector may be viewed as a continuous string of directional reflectors. The scattering pattern of the array of wires can be obtained from that of a single wire by multiplication with an appropriate array factor.

The current theory of the distortion regarding course information due to wheel-shaped barbedwires fences has been proved by computer-based simulation and experimentation compare to mathematical analysis. Finally the circular barbed-wires effects must be taken into account.

\title{
References
}

[1] U. S. Department of Transportation Federal Aviation Administration, Order 6820.7B Maintenance of navigational aids facilities and equipment, (1988).

[2] U. S. Department of Transportation Federal Aviation Administration, Order 6820.9 VOR, VOR/DME, VORTAC Installation Standard Drawings, (1988).

[3] U. S. Department of Transportation Federal Aviation Administration, Order 6820.10 VOR, VOR/DME, VORTAC Siting Criteria, (1988).

[4] S. Odunaiya and D. Quinet, "Calculations and analysis of signal processing by various navigation receiver architectures", Avionics Engineering Center, Ohio University, (2004), pp.1.D.1.8-12.

[5] H. C. Hurley, S. R. Anderson and H. F. Keary, "The CAA VHF omnirange", Proc. IRE, (1951).

[6] S. R. Anderson and R. B. Flint, "The CAA VHF omnirange", Proc. IEEE, (1959).

[7] K. Yamamoto and M. Nagaoka, "A Bearing error in the VHF omnirange due to sea surface reflection", IEEE Transactions on antennas and propa- tion, vol. 2, (1989), pp. 181-185.

[8] H. Gruenberg, "Bearing Error in the VHF omnirange due to Scattering from wires", IEEE Transactions on antennas and propagation, vol. AP-26, (1978).

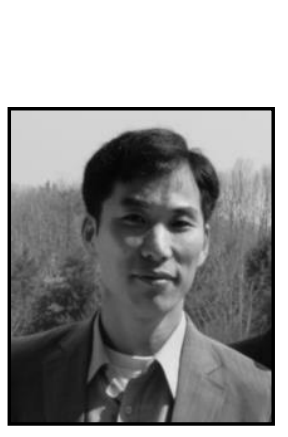

\begin{abstract}
Authors
Jeong il Moon, he received his B.S. degree in Physics from the University of Suwon in 1995, and Computer Science from the Korea open university in 2004. Master of Engineering degree from the National Gangneung-wonju University in 2010. He is enrolled in a doctoral course at the Korea National University of Transportation in the Department of Computer Engineering. He is a faculty member of Civil Aviation Training Center, KAC from 2009. From 1995 to 2008 he was a engineer in KAC. His research interests are in the areas of air navigation system, radio communication, and receiver of these methods to air navigation system.
\end{abstract}

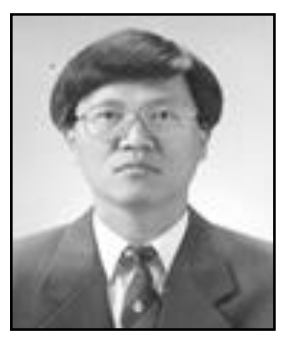

Yoonsik Kwak, he received his B.S. degree in Electrical Engineering from the University of Cheongju in 1984, his M.S.E.E. degree from the University of Kyunghee in 1986 and his Ph.D. degree from the University of Kyunghee in 1994. He worked at Korea National University of Transportation in the Department of Computer Engineering and rose to the level of Full Professor. His research interests are in the areas of signal processing, Internet communication, microcomputer system, and applications of these methods to mobile system. 
International Journal of Control and Automation Vol. 8, No.4 (2015) 